\title{
The use of neighbourhood intensity comparisons, morphological gradients and Fourier analysis for automated precipitate counting \& Pendellösung fringe analysis in X-ray topography
}

\author{
G. Murphy ${ }^{1}$, P. F. Whelan ${ }^{1}$, P. J. McNally ${ }^{2, a}$, T. Tuomi ${ }^{3}$, and R. Simon ${ }^{4}$ \\ 1 Vision Systems Group, Research Institute for Networks \& Communications Engineering (RINCE), Dublin City University, \\ Dublin 9, Ireland \\ 2 Microelectronics Group, RINCE, Dublin City University, Dublin 9, Ireland \\ 3 Optoelectronics Laboratory, Helsinki University of Technology, PO Box 3500, 02015 TKK, Espoo, Finland \\ ${ }^{4}$ Institute für Synchrotronstrahlung (ISS), Forschungszentrum Karlsruhe GmbH, Postfach 3640, D-76021 Karlsruhe, Germany
}

Received: 30 June 2003 / Accepted: 31 December 2003 - (C) EDP Sciences

\begin{abstract}
Crystal distortions modify the propagation of X-rays in single crystal materials, and X-ray topography can be used to record these modifications on a film thus providing images of the distributions and nature of defects, dislocations, strains, precipitates, etc. in semiconductors. Small variations of contrast, which often need to be analysed can be rendered invisible. Furthermore, artefacts in the films must be removed. This study examines the use of advanced image analysis techniques applied to a selection of X-ray topographs in section transmission mode: (i) the automated counting of oxygen-related precipitates and (ii) the enhancement of Pendellösung fringes. The technique also succeeds in removing unwanted features in the original x-ray topographs such as vertical streaking due to collimating slit phase contrast and strain features near the surface due to the presence of integrated circuit process strains.
\end{abstract}

PACS. 07.05.Pj Image processing - 61.72.Ff Direct observation of dislocations and other defects (etch pits, decoration, electron microscopy, x-ray topography, etc.) - 81.05.-t Specific materials: fabrication, treatment, testing and analysis

\section{Introduction}

X-ray topography (XRT) is a non-destructive technique, which can provide detailed information on defect distributions in crystals and is mainly used for the study of dislocations, planar defects, stacking faults, domain walls in ferromagnetic materials, growth defects or large precipitates [1-3]. Analysis of the topographs is often difficult since many intricate features appear in a single image. The topographs often present small variations of contrast and areas corresponding to very high or very low densities of grey can be quite invisible. Artefacts are created due to film damage, phase contrast effects due to dust on collimating slits, scratches on samples and recording films, etc. [4]. This paper examines two different imaging problems related to two different types of topograph: (A) the identification, segmentation and counting of oxide-related precipitates which appear as dark blobs of varying intensity and shape in an image and (B) enhancing and highlighting Pendellösung fringes, which appear as black and white horizontal lines, while removing unwanted vertical and diagonal streaks and artefacts.

\footnotetext{
a e-mail: patrick.mcnally@rince.ie
}

\section{Experimental}

The measurements were performed at the HASYLAB am DESY Synchrotron, Hamburg, Germany as described elsewhere $[5,6]$ and at the ANKA Synchrotron, ISS, Karlsruhe, Germany. In section topography, which was used in this study, the beam was limited by a horizontal slit having a width of $15 \mu \mathrm{m}$, and the (001) silicon wafer surface was at an angle of $20^{\circ}$ with respect to the incident beam. The images were then generated from the films by using a CCD camera in conjunction with an optical microscope and these images are saved in 'tif' format. Analysis of the images was carried out in NeatVision ${ }^{\circledR}$ (www.neatvision.com) which is a Java-based computer vision package developed by the Vision Systems Group at DCU.

\section{Automated counting of images of oxygen-related precipitates in section transmission topographs of a silicon wafer}

Section transmission topography results in nondestructively acquired images of a "slice" of a 
semiconductor wafer, in the case of this study, heattreated Czochralski-grown $\mathrm{Si}$ wafers. The wafers are (100) oriented p-type $\mathrm{Si}$ wafers, with a resistivity of 8-15 Ohm-cm. They were subjected to a $1160{ }^{\circ} \mathrm{C}$ anneal in a $\mathrm{N}_{2}$ ambient in order to simulate typical thermal annealing conditions in an IC process, which has been described previously [5]. When the Si wafer is subjected to thermal cycling during production, the presence of oxygen impurities in the wafer results in the nucleation of large oxygen-related precipitates. These appear as black "blobs" in the section transmission topographs, and their presence may be related to inferior electrical performance [6]. An original unprocessed section topograph (ST) of a Si wafer is shown in Figure 1. The spatial

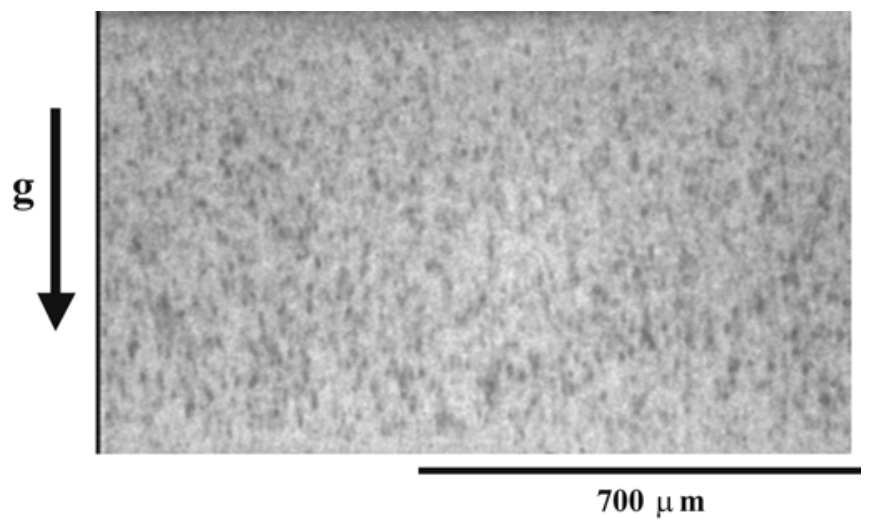

Fig. 1. Typical 220 section topograph showing precipitates in a $\mathrm{Si}$ wafer. The projection of the diffraction vector $\mathbf{g}$ in the plane of the recording film is indicated.

resolution in X-ray topography is $1-2 \mu \mathrm{m}$ and the spatial resolution on the film is approx. $1 \mu \mathrm{m}$. A 256 (8-bit) grey-scale intensity range was used to manipulate data in the NeatVision environment. One of the main difficulties in counting precipitates lies in the fact that they vary in size, shape and intensity (the precipitate images can vary in size from 1-50 $\mu \mathrm{m}$, though one must count them all within a typical topograph).

The problem of counting the precipitates in a topograph is resolved into three main parts, namely, identification, separation and finally counting. A selected $\sim 50 \mu \mathrm{m}$ $\times 50 \mu \mathrm{m}$ region of the original section topograph is shown in Figure 2(a). The use of regional maxima [7] and various neighbourhood patterns are two methods, which were assessed to identify the precipitates. Also, overlapping precipitate images must be separated. This was achieved through using a combination of morphological gradient generation and conditional dilation. This process is now outlined.

Due to the illumination gradient inherent in the CCD capture process, simply identifying the precipitates (such as those illustrated in Figure 2(a)) using a global threshold does not work. This produces results where there are significantly more precipitates at the edges of the images than in the centre. There are a number of methods to remove illumination gradients such using as black or white
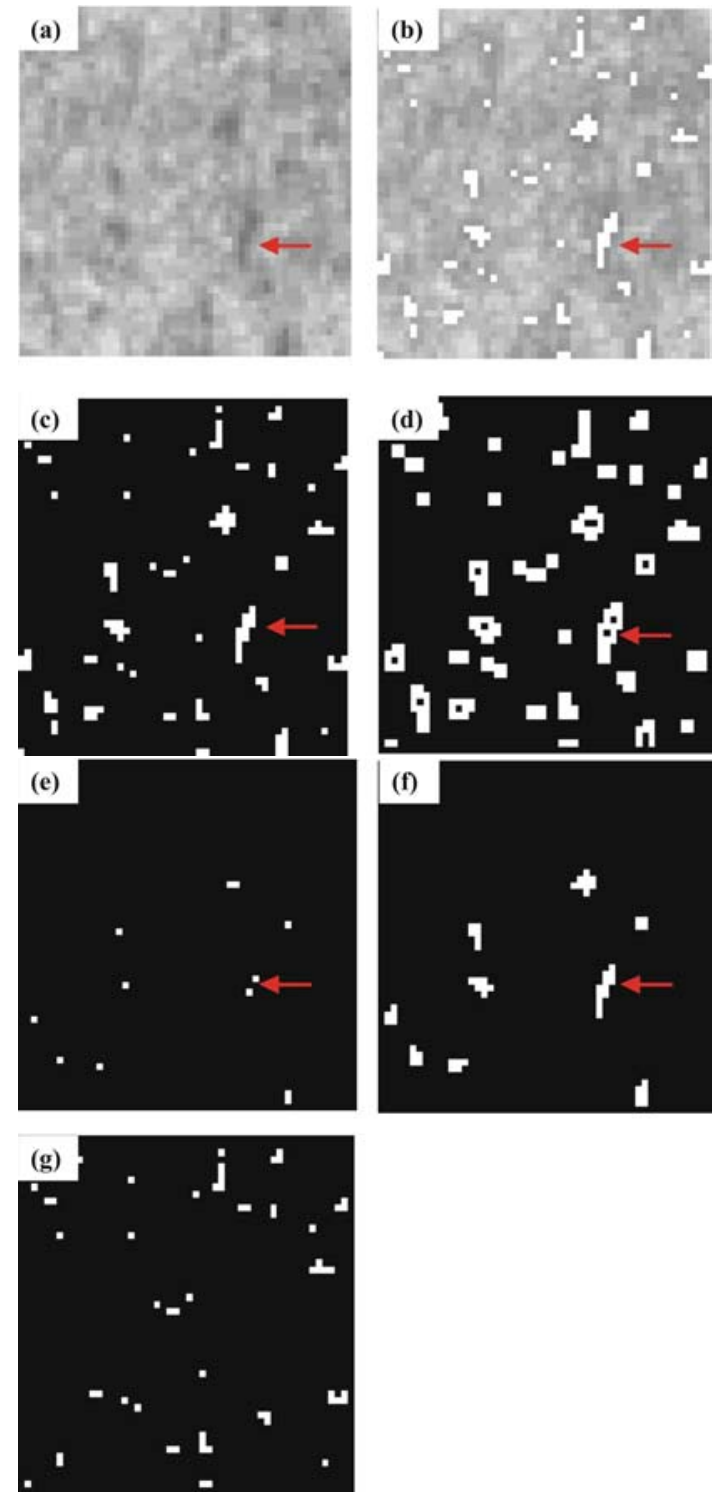

Fig. 2. Automated image analysis of the precipitate images. (a) Section from a topograph showing the precipitates. (b) Identified precipitates (in white) overlaid on the original image. (c) Mask image: thresholded version of image (b). (d) Morphological gradient of image (c). (e) Marker image: result of subtracting image (d) from image (b). (f) Conditional dilation of the marker image using the binary mask. (g) Subtraction of the conditionally grown image from the mask image.

"top hats" [7-10]. Using this methodology, some of the precipitate information can be lost as it can be identified incorrectly as noise.

For the identification of the precipitates a number of different neighbourhood patterns were compared. In each case the intensity of the central pixel was found and compared to the average of the outer pixels in the neighbourhood. If a sufficient difference exists between the two values then that pixel was copied to the output image as it formed either a part of a blob or an entire blob. All other pixels were transformed to white in the output 
image (Figure 2(b)). The resulting image was thresholded (Figure 2(c)) and is referred to as the "mask" image for conditional dilation. The thresholding operation performs two functions: it creates a binary image where the precipitates are represented as white blobs on a dark background in preparation for counting and also by performing a logical "OR"-ing of this image with the original image, the locations of the precipitates are highlighted.

The edge pixels were used rather than the complete neighbourhood due to the variable but small size of the precipitates. This is not an issue as the number of precipitates is small compared to the height and width of the image. It was found that the $7 \times 7$ square neighbourhood pattern containing 24 boundary pixels gave the best results.

A major difficulty lies in identifying the precipitates that tend to touch and overlap (an arrow points out once case of overlapping blobs in Figure 2(b)). Precipitate images should ideally be circular due to the spherically isotropic stress distribution surrounding them [11]. Hence any elongated images are most likely due to overlapping precipitate images and these precipitates need to be separated. A morphological gradient [10] outlines the overlapping images and a very small structuring element $(2 \times 2$ array) is required due to the small size of the precipitate images (Figure 2(c)). When the morphological gradient is found (Figure 2(d), note the boundary around the two overlapped blobs), it is subtracted from the original image. This leaves a marker for each of the overlapped blobs and also for any of the larger blobs, this image is referred to as the "marker" image (Figure 2(e)) These markers are counted and the value stored. Using the original image as a mask, the marker image is grown by conditional dilation (Figure 2(f)) using the mask image generated previously (Figure 2(c)) to condition the growth process. The resultant image contains the precipitates, which the morphological gradient has highlighted. This is subtracted from the original image (as these have already been counted and segmented) resulting in the image illustrated in Figure 2(g). The remaining non-overlapping precipitates are then counted. Both counts are then added to give the total number of precipitates in the image.

A dilation count method was used, as it counts all the identified blobs with $100 \%$ accuracy regardless of orientation and shape. The algorithm is as follows: (a) raster scan the image until a white pixel is found, (b) increment counter, (c) copy white pixel to a black holding image of the same size as the original, (d) use the holding image as a marker and the original image as a mask and grow the blob by conditional dilation (10 iterations), (e) subtract this image from the first image (thus removing the blob from the image), (f) keep repeating the five steps above until the raster scan of the image is completed. This method will accurately count all 4-connected precipitate images regardless of shape, size of orientation. A complete topograph with approximately fifteen hundred precipitates takes of the order of 1 minute on a standard personal computer. Experimentation has shown that the dilation

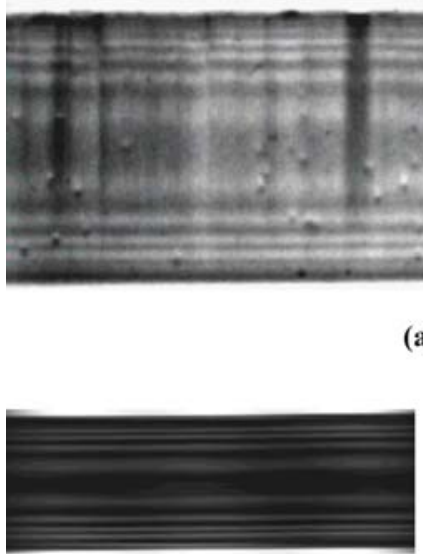

(b)

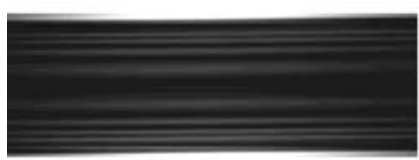

(d) (a)

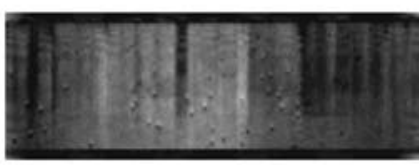

(c)

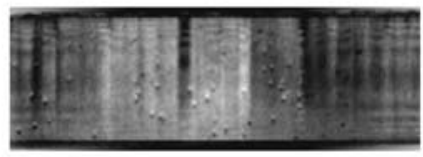

(e)
Fig. 3. Images after processing and images of the removed noise. (a) Original unprocessed 220 section transmission topograph image of a Si wafer displaying Pendellösung fringes, oxygen-related precipitates and vertical streaks; (b) after the inverse Fourier transform was performed on an image which has had a 5-pixel wide strip pass filter applied; (c) the noise comprising of uneven illumination and streaks which has been removed; (d) after the inverse Fourier transform was performed on an image which has had a 1-pixel wide strip pass filter applied; (e) the noise comprising of uneven illumination and diagonal streaks which has been removed.

count method matches the actual count for a wide range of different regions within our test topograph's dataset.

\section{Enhancement of images of Pendellösung fringes (PFs) in section transmission topographs of nearly perfect silicon}

An evaluation of the relative crystalline perfection of the Si substrate is one of the crucial factors impacting the reliability of completed ICs. This evaluation can be carried out using X-ray topographic techniques, where a particular emphasis can be placed on the appearance (high crystalline quality) or disappearance/perturbations of Pendellösung fringes (strained or low quality) in Si substrates [13-17]. An example of the Pendellösung fringe $(\mathrm{PF})$ phenomenon is shown in Figure 3(a), which is a 220 X-ray transmission section topograph of a section of a silicon wafer. Other unwanted features include vertical streaks due to the effect of the phase contrast impact of dust, or imperfections in the collimating slits. Backside damage can lead to vertical and horizontal streaks and is often due to scratching and mechanical damage of the back 
of the wafer. These must be removed. Oxygen related precipitates are also observed as the small black/white blobs throughout the topograph image. Another problem is uneven illumination of the image.

Due to the periodic nature of the information in the images, and in light of previous research, it appears that Fourier analysis could be an effective method for solving this problem. However, the low pass spatial filtering methodology of [4] cannot be directly used for PF enhancement. Firstly, the brightness gradient must be removed. The authors used a mathematical morphological white "top hat" (image minus opening with a structuring element), and $11 \times 11$ and $21 \times 21$ sized structuring elements (SE) [7-10]. It was found that opening with an $11 \times 11$ SE identifies the PFs as a background and tries to remove them. However, while the resultant images are darker, the lighting gradient is removed and the images are more uniform. Removing the brightness gradient with a white top hat using a $21 \times 21 \mathrm{SE}$ gives the best results.

The PFs are separated from the image noise by the application of the Fourier transform [7-9]. Examining the magnitude image, after applying the Fourier transform, both as an image and as a thresholded image did not show any obvious peaks in the spectrum relating to the vertical and/or any diagonal streaks. However, the required information contained in the PFs exist in the horizontal plane of the original image. Thus in frequency space, all the information of interest exists in the vertical plane. In order to isolate the information in the vertical plane, a strip pass filter was used. This filter only passes information contained in the vertical axis out to a certain number of pixels on either side. The impact of using the Fourier transform with a strip pass filter [7-9] to efficiently highlight the PFs is shown in Figures $3(\mathrm{~b})-(\mathrm{e})$. It can be seen in Figure 3(d) that when the filter is one pixel wide, information at the left and right edges of the original image is lost. This is most easily observed in Figure 3(e) which is an image of what has been removed from the image, i.e. the brightness gradient, which was removed with a white top hat and the diagonal or vertical streaks and backside damage. Excessive information tends to be removed, and this is particularly acute near the left- and right-hand upper surfaces. This tends to lead to less definition of the PFs near the upper and lower surfaces of the topograph. However when a strip pass filter of five pixels wide is used then the information at the edges is preserved; see Figures 3(b)-(c) for comparison. Successively adding the resultant image to the original image emphasises the PFs and de-emphasises the noise in the image. This acts as a quick check that the horizontal black and white lines found are indeed the Pendellösung fringes.

\section{Conclusions}

\subsection{Precipitate counting}

The results have shown that electronic image processing and identification of oxygen-related precipitates is achieved using a seven by seven-square neighbourhood.
The overlapping precipitates are correctly separated out using a morphological gradient. The precipitates have been accurately counted regardless of orientation, shape or size by a counting algorithm based on mathematical morphological methods.

\subsection{Pendellösung fringe enhancement}

Removing the brightness of the gradient of the image must be the first pre-processing step. This is performed via mathematical morphological methods. The Pendellösung fringes are then separated from the unwanted streaks or noise in the image by strip pass filtering the magnitude image in the Fourier domain. It was found that using a filter with a width of five pixels produced the best results.

This work was supported by the IHP-Contract HPRI-CT-199900040/2001-00140 of the European Commission. The authors thank C. Paulmann at beamline F-1, HASYLAB, Germany.

\section{References}

1. A. R. Lang, Diffraction and Imaging Techniques in Material Science, edited by S. Amelinckx, R. Gevers, J. Van Landuyt (Amsterdam: North-Holland, 1978), p. 623

2. T. Tuomi T, K. Naukkarinen, P. Rabe, Phys. Stat. Sol. A 25, 93 (1974)

3. A. Authier, Diffraction and Imaging Techniques in Material Science, edited by S. Amelinckx, R. Gevers, J. Van Landuyt (Amsterdam: North-Holland, 1978), p. 715

4. M. Pilyard, Y. Epelboin, A. Soyer, J. Appl. Cryst. 28, 279 (1995)

5. P. J. McNally, J. W. Curley, M. Bolt, A. Reader, T. Tuomi, R. Rantamäki, A. N. Danilewsky, I. DeWolf, J. Mater. Sci.: Mater. Electron. 10, 351 (1999)

6. T. Tuomi, M. Tuominen, E. Prieur, J. Partanen, J. Lahtinen, J. Laakkonen, J. Electrochem. Soc. 142, 1699 (1995)

7. P. F. Whelan, D. Molloy, Machine Vision Algorithms in Java, Techniques and Implementation (Springer-Verlag, 2001)

8. P. Soille, Morphological Image Analysis: Principles and Applications (Springer-Verlag, 1999)

9. J. C. Russ, The image processing handbook (CRC Press, 1992)

10. L. Vincent, IEEE Trans. Image Proc. 2, 176 (1993)

11. L. Ming, Z. H. Mai, S. F. Cui, Acta Cryst. A50, 725 (1994)

12. S. Beucher, F. Meyer, Image Processing, edited by Dougherty (Marcel Dekker Inc., 1993), pp. 433-483

13. P. J. McNally, A. N. Danilewsky, J. W. Curley, A. Reader, R. Rantamäki, T. Tuomi, M. Bolt, M. Taskinen, Microelectron. Eng. 45, 47 (1999)

14. E. S. Meieran, I. L. Blech, J. Appl. Phys. 36, 3162 (1965)

15. Y. Epelboin, J. Appl. Phys. 64, 109 (1988)

16. D. K. Bowen, B. K. Tanner, High Resolution X-Ray Diffractometry and Topography (Taylor \& Francis, London, UK, 1998)

17. J. Partanen, T. Tuomi, J. X-Ray Sci. Technol. 2, 165 (1990) 